\title{
Gel filler dermotension
}

\section{Marlen Andreevich Sulamanidze, Alexander Ivanovich Nerobeyev, Arnold Aramovich Adamyan, George Marlenovich Sulamanidze, Konstantin Marlenovich Sulamanidze}

Clinic of Anaplastic and Aesthetic Surgery TOTALCHARM, Moscow, Russia - Tbilisi, Georgia. A.V. Vishnevskiy Institute of Surgery. Russian Academy of Postgraduate Medical Education, Moscow, Russia

\section{Email address:}

gracia@aptos.ru (M. A. Sulamanidze)

\section{To cite this article:}

Marlen Andreevich Sulamanidze, Alexander Ivanovich Nerobeyev, Arnold Aramovich Adamyan, George Marlenovich Sulamanidze, Konstantin Marlenovich Sulamanidze. Gel Filler Dermotension. Journal of Surgery. Vol. 2, No. 5, 2014, pp. $72-77$. doi: $10.11648 /$ j.js.20140205.14

\begin{abstract}
Introduction. During a classical dermotension operation usually skin incision is usually performed, a cellulocutaneous flap is mobilized, i.e. a subcutaneous pocket is created wherein an expander is inserted. Then the wound is closed and the process of filling the elastic bulb begins after the stage of stable wound cicatrization being reached. Material and Methods. A new dermotension method is proposed, which is essentially a creation of a subcutaneous cavity by means of subcutaneous dissection without incisions, through punctures, with subsequent gradual stretching of the skin area with the help of the gel component. Results. Since 1995 the gel dermotension method has been applied in 33 cases to the areas of the face, upper/lower limbs and the trunk of the body. The operations were performed effortlessly and quickly occasionally followed by a slight post-operational syndrome noticed. The dermotension process began on the first day. A sound plastic material thus achieved was used for its designated purpose on the second stage. Conclusions. The presented method is cost-effective, the first stage is minimally invasive while the dermotension process is accelerated by 12 to 14 days.
\end{abstract}

Keywords: Dermotension, Subcutaneous Dissection, Gel Component, Minimally Invasive Method

\section{Introduction}

In dermatoplastic surgery local flaps grafting is routinely preferred due to a number of advantages over other plastic surgery techniques. However, it is always limited by the amount of plastic material available and is associated with a specific set of indications.

In 1976 Radovan reported a new method of tissue augmentation subsequently dubbed the tissue expansion method, or balloon dermotension, which diversified the range of indications for local flaps grafting1.

Over the recent years quite a few issues have been settled concerning laying down indications and counter-indications, prevention of complications, expansion conditions, characteristics of tissue extension.

At the same time, the shortcomings have also been identified: the need for preliminary skin incision while creating an expander socket and associated bleeding, marked post-operational pain syndrome, the need for sewing the wound and waiting till it closes which extends the dermotension process by 12 to 14 days. Moreover, the cicatrix produced by such incision and the damaged skin in the port area reduce the eventual area of plastic material2.

The purpose of this account is to provide a new dermotension technique free from the abovementioned disadvantages and making use of the possibilities to create subcutaneous cavities to be filled with liquid or gel. We have dubbed the suggested technique the "liquid-gel dermotension"3,4.

\section{Material and Methods}

The method is based on applying a special device, a wire scalpel (Aptos Wire), and a corresponding method of subcutaneous soft tissue dissection, through cutaneous punctures, as well as using a gel component for gradual stretching of the skin. ${ }^{2,3}$ The Aptos Wire consists of a double-edged needle up to $10 \mathrm{~cm}$ long and a steel multiplestranded cerclage wire $(2 / 0$ or $3 / 0)$ up to $50 \mathrm{~cm}$ long attached to it (Fig. 1).

A skin area which dermotension would be applied to was singled out for a subcutaneous cavity to be created near the defect. After anesthesia applied the needle was stuck into the skin and inserted into the subcatenous fat at the required depth. 
Then the needle was moved along the edges of the detachment zone. If necessary, the needle was put out at the zone contour bends, brought to the surface (but not completely so) with the second edge remaining above the skin, so the progression of the needle was continued using the second edge. The entire designated area thus marked, the needle and the wire were introduced into the first insertion point. Holding and pulling the wire ends, we dissected the subcutaneous tissues with smooth sawing movements thereby creating a cellulocutaneous flap, or subcutaneous cavity.

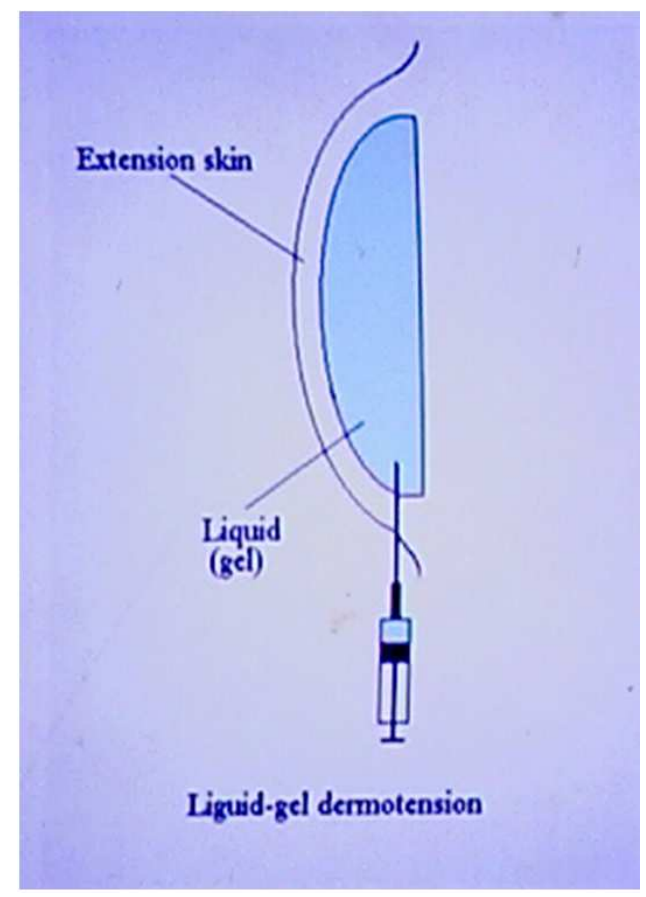

Fig 1. Diagram of the gel dermotension process.

Special attention was paid to the depth of cellulocutaneous layer dissection, the anatomical features of a specific area, its blood supply which helped avoiding bleeding and hematomas. The manipulation itself was effortless, quick, and painless.

In the immediate wake of the operation the gel was injected into the cavity until it was tightly filled, henceforth continuing the skin expansion process with the help of the gel. The intervals between filler insertions were identical to those for other types of tissue expansion and equal to 2-5 days (Fig. 2) (see Video at).

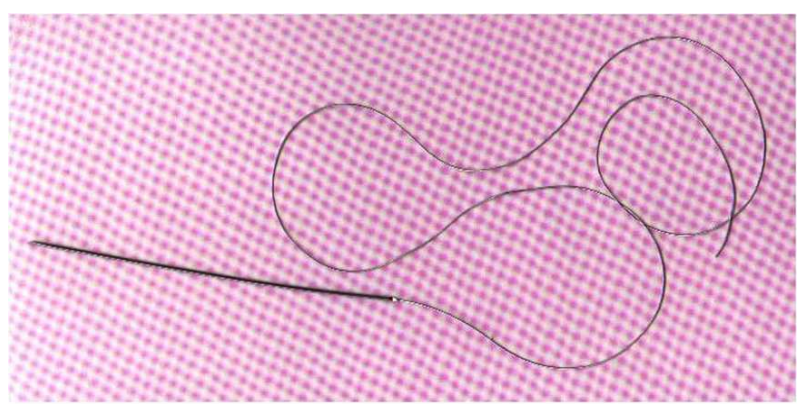

Fig 2. Wire Scalpel

\section{Results and Discussion}

The gel dermotension method was first used in 1995. Since then this kind of surgical intervention has been performed in 33 operations on the face, upper/lower extremities and the trunk. On each occasion the operation of creating a subcutaneous cavity was performed effortlessly and quickly with an occasional slight post-operational syndrome; dermotension was applied as soon as the subcutaneous cavity was created, i.e. 10 to 14 days earlier than in the case of expander dermotension. During the subsequent period the gel insertion procedure was accompanied by slight pain which disappeared without any external assistance within a few hours. The cutaneous expansion was continued until the desired size of the expanded skin area had been achieved.

After the dermotension was completed, at the second stage of the operation (after the removal of the gel filler) it was noted that the internal surfaces of the flap, as well as those of the donor surface, were smooth and covered with a thinwalled capsule; the flaps expanded well and adapted adequately to the new location. ${ }^{4}$

The 3/0 Aptos Wire was applied to the face and neck areas, i.e. to the areas where skin and subcutaneous fat is thin and soft while a device with a stronger and thicker wire $(2 / 0)$ was used in other areas (body, superior and inferior limbs).

\section{Case History}

Patient K, 24 years old, visited the clinic with a complaint for an atrophic sagittal cicatrix covering the entire surface of the dextral crus and part of the knee, which created a distinct aesthetic deformation of the right lower limb.

Using the subcutaneous dissection method a single cavity of a complex shape was formed in the patellar and knee areas. The cavity was then filled with $230.0 \mathrm{ml}$ of gel. 35 to $40 \mathrm{ml}$ of filler were daily inserted into the created cavity every 2 to 4 days (a total of $580 \mathrm{ml}$ ). 24 days later a good level of skin regeneration was achieved and the second stage operation was performed (Fig. 3,4,5).

\section{Case History 2}

Patient B., 24 years old, was admitted to the Semashko Railway Hospital on 19.06.96 with complaints of a coarse skin cicatrix in the Achilles tendon area of the left lower extremity, frequent ulcerations, intolerance to standard footwear. Anamnesis: 2 years earlier due to traumatic Achilles tendon rupture a tendon restoration surgery was performed. The wound healed by secondary intention. Corresponding surgical and therapeutic treatment was implemented, none to any effect.

An operation according to the described technique was conducted in our clinic (during 17 days, using $160 \mathrm{ml}$ polyacrylamide gel). The second stage included incising the cicatrix and closing the wound with an extended flap. Postoperational period revealed no complications, re-examination performed in 4 months' time (6-12). 


\section{Case History 3}

Patient D., 23 years old, came to Cosmetic Medicine Centre of the Central Clinical Hospital of Russian Academy of Sciences on 01.11.96 with complaints of vicious cicatrices on forehead and face.

Anamnesis: the patient had suffered a heavy injury in a water scooter accident.

Objectively observed: hypertrophic cicatrices in the forehead, brow, upper eyelid, zygomatic (left side) areas, lateral part of the brow $3-4 \mathrm{~cm}$ below its natural position, in the temporal region (12).

Dermotension was performed in accordance with the suggested technique. Total biogel injected since the beginning of the operation - $92.0 \mathrm{ml}$, sufficient quantity of quality plastic material obtained in 16 days.

The second stage included incising the cicatrix and draining the cavity. A red-tinted gel without visible changes in consistency and other properties was thus obtained, bacteriological analysis proving it to be sterile.

Post-operational period revealed no complications, wound healed by primary intention, stitches removed on day 12 after operation. The patient revisited our clinic 18 years later for other aesthetic manipulations and we were able to take a picture of her (15).

Pictures of other clinical cases are also included: Fig. 6-15.

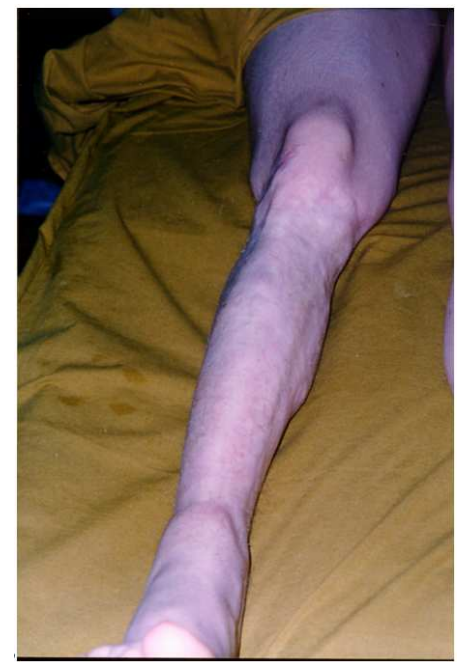

Fig 3. Patient K Cicatricial deformity of the right crus (lower leg).

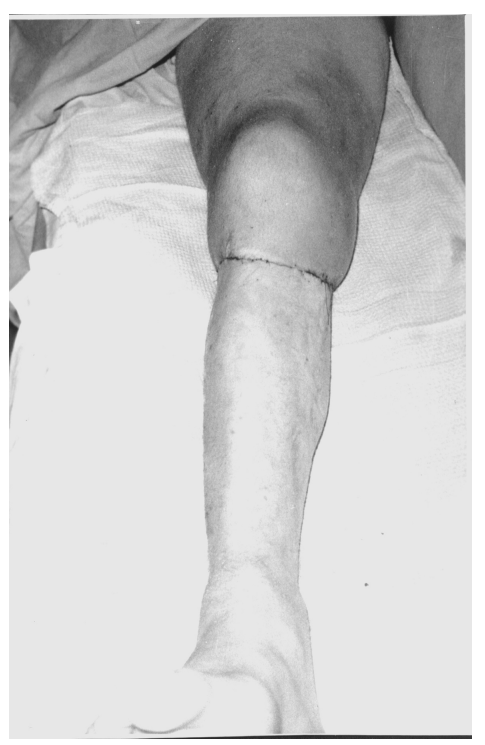

Fig 4. Patient $K$ Condition after cutaneous expanded flap surgery (at the stitch removal stage).

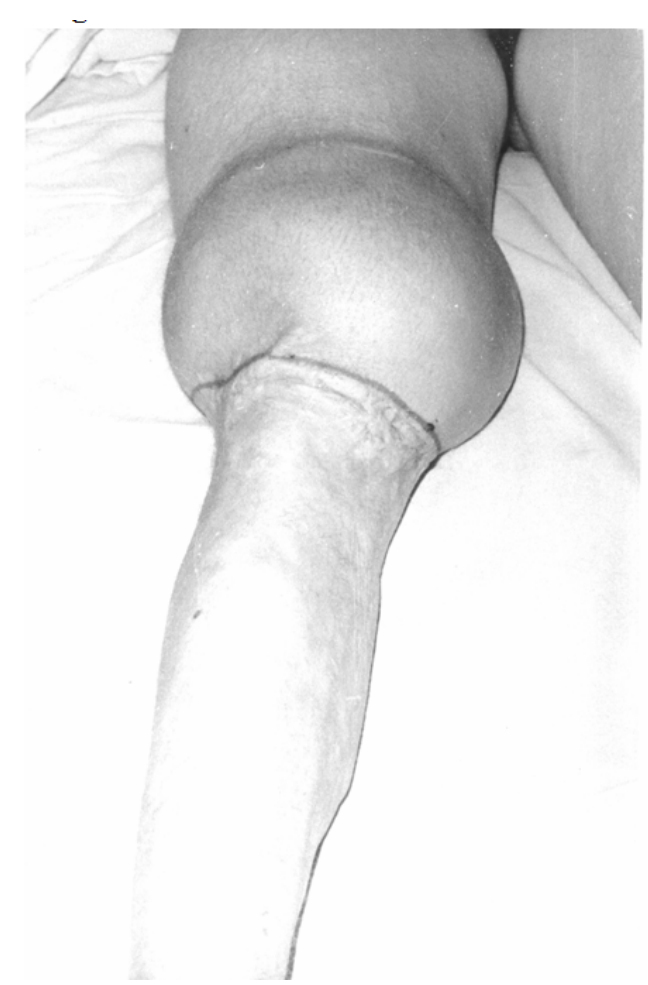

Fig 5. Patient K Subcateneous cavity formed above the defect. Gel insertion over a 21 days' period. A good level of skin regeneration achieved.

Pictures of other clinical cases are also included: Fig. 6-15. 


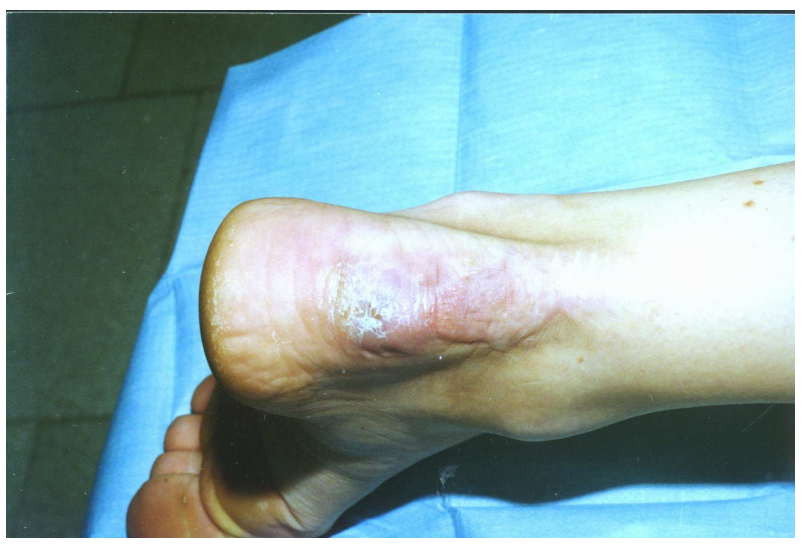

Fig 6. Patient B Rough vicious cicatrix in the Achilles tendon area.

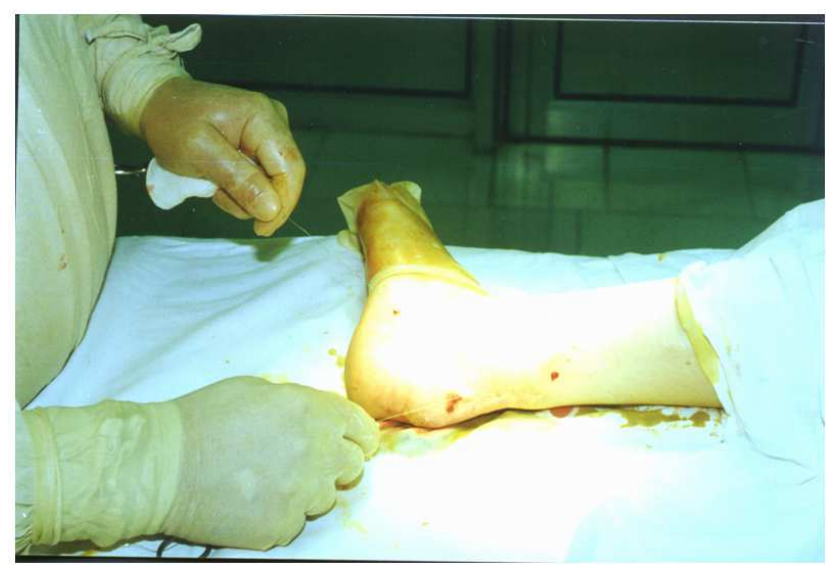

Fig 7. Patient B Cavity formation stage (using the Wire Scalpel).

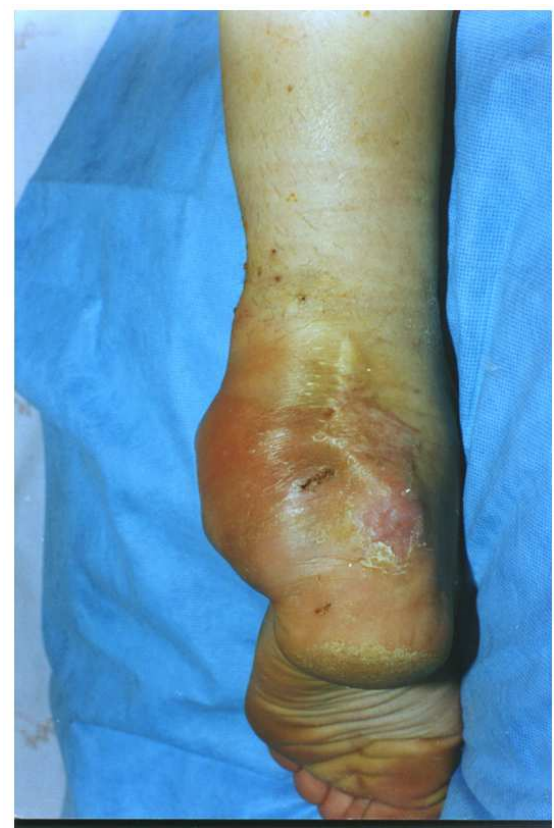

Fig 8. Patient B Condition after 17 days - flap expanded

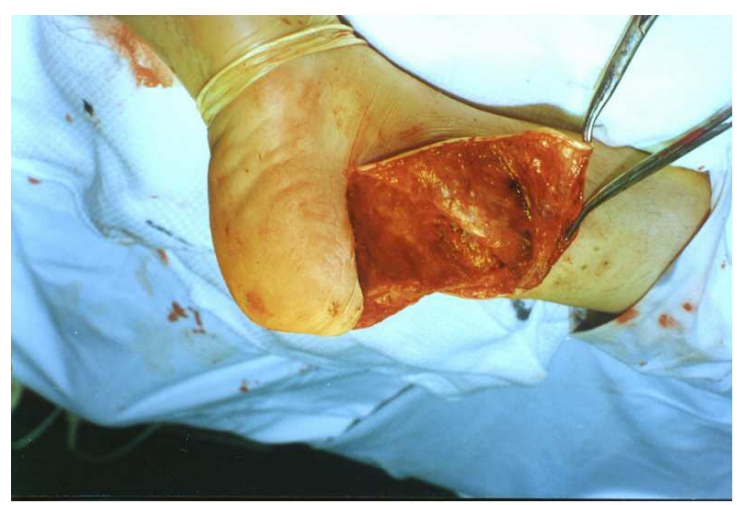

Fig 9. Patient B After removing the gel and excision of cicatrix, the flap that was placed in the defect area is considered to be of good quality.

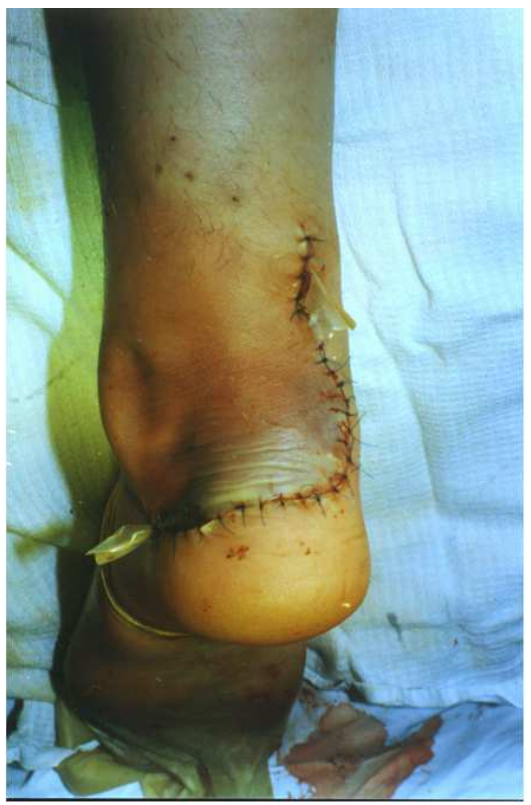

Fig 10. Patient B Wound closed

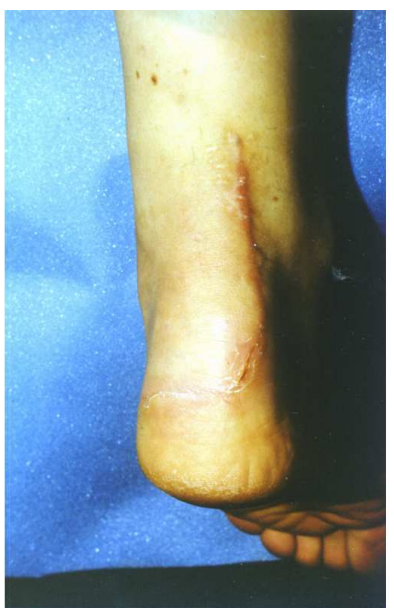

Fig 11. Patient B After 4 months. 


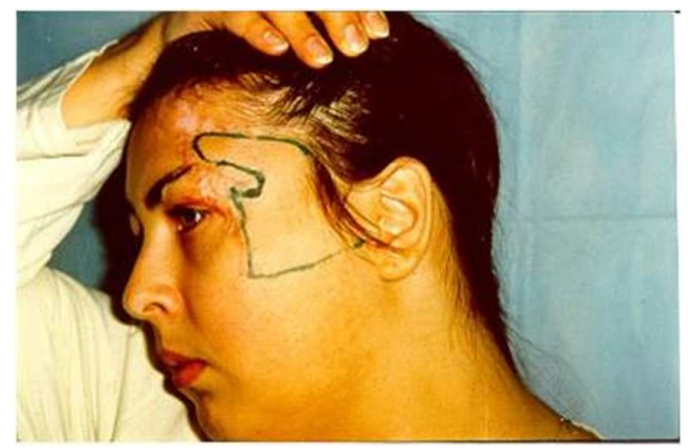

Fig 12. Patient D Dermotension applied on a patient with rough vicious cicatrix in the left-sided frontotemporal paraorbital region. Note that a cavity of an irregular form was cut which made it impossible to use a mass produced expander.

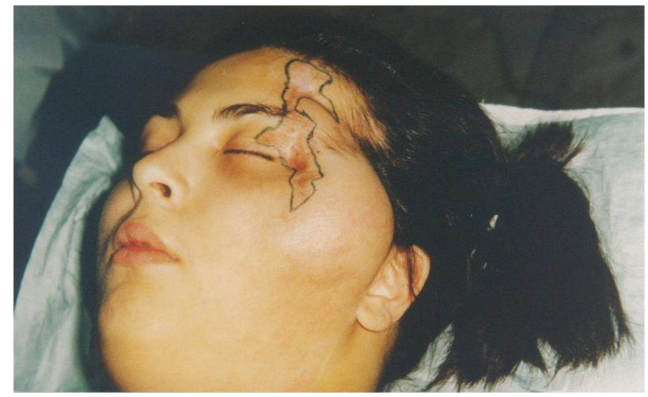

Fig 13. Patient D 17 days after stage 1 of the operation a sufficient cutaneous expansion was achieved.

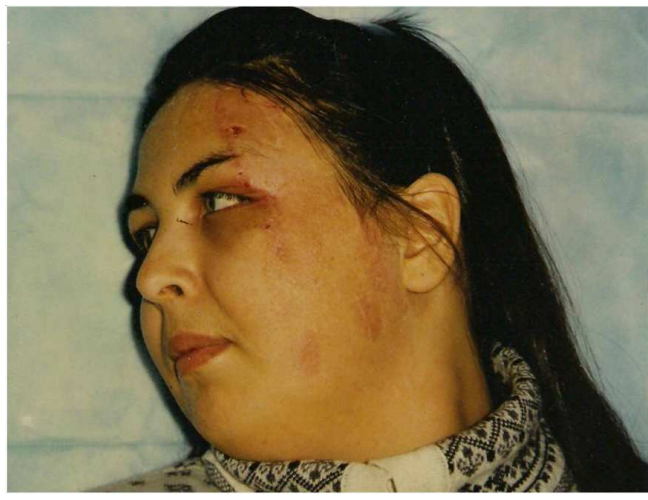

Fig 14. Patient $D 10$ days after expanded flap surgery of the cicatrix.

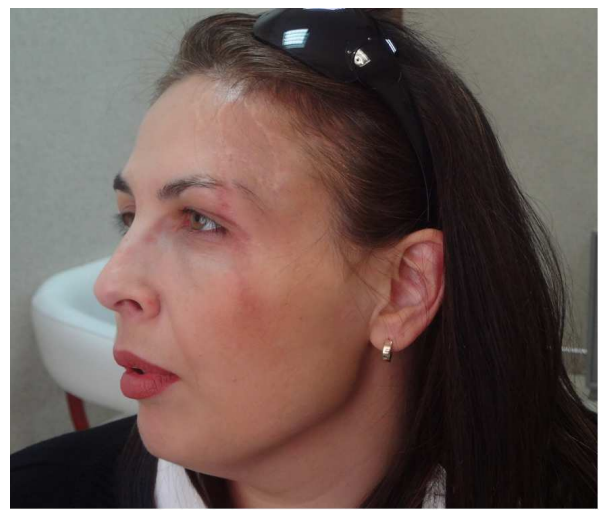

Fig 15. Patient D 18 years after operation.

\section{Conclusions}

1. The new dermotension technique is notable for being straightforward and does not require such an expensive device as expander.

2. Due to absence of external incisions the operation is less traumatic, post-operational pain syndrome is insignificant, risk of complications declines, there is no need to suture the wound and waiting for cicatrization thereof which is why the dermotesion process begins from day 1 and is shortened by 12-14 days.

3. A possibility appears to create subcutaneous cavities and henceforth cellulo-cutaneous flaps of any shape and size. In addition, dermotension can be performed in critical areas of the body.

4. Due to absence of cicatrices and damaged skin in the areas of filler injection - as is often the case in the area of the expander port in balloon dermotension - the size of the flap is smaller and easier to handle.

5. The liquid-gel dermotension allows obtaining rigorous plastic material hardly inferior and, indeed, in some cases, superior in certain characteristics to the material obtained by other types of tissue expansion.

\section{Disclosures}

Drs. Marlen Sulamanidze and Aleksandr Nerobeyev have been holders of the Russian patents on the Wire Scalpel and Liquid-Gel Dermotension method since 1995.

Drs. Marlen, George and Konstantin Sulamanidze are coowners of the Aptos Company which manufactures and distributes wire scalpels (Aptos Wire).

\section{Funding}

The authors received no financial support for the research, authorship, and publication of this article.

The Article elaborates on:

1. The wire scalpel device (Aptos Wire) applied in plastic, reconstructive and aesthetic surgery for undercutting (dissection) of the skin integuments without making external incisions, through punctures.

2. Formacryle - polyacrylamide gel manufactured by Moscow Mechanical Rubber Goods Factory used in various integumentary implant (mammary, testicular) filling, etc. Similar gels are manufactured in Ukraine (Interfal) and Italy (Aquamid).

3. Plasmagel - blood plasma fraction that underwent special heat treatment $\left(120{ }^{\circ} \mathrm{C}\right.$ over $15-20$ minutes $)$. Plasmagel is produced ex tempore prior to injection, or in advance from the blood plasma of the patient or donor.

\section{References}

[1] Radovan C. Tissue expansion in soft-tissue reconstruction. Plastic and Reconstructive Surgery, 74. 482 (1984). 
[2] Sulamanidze M.A., Nerobeyev A.I., Vozdvizhenskiy I.S. Tissue expansion techniques. Annals of Surgery, Issue No.2, Moscow, 1997, pp. 64-71.

[3] Sulamanidze M.A., Britun Yu.A., Savchenko S.V. Subcutaneous dissection and liquid-gel dermotension. Annals of Plastic, Reconstructive and Aesthetic Surgery, Issue No.2, Moscow, 1997, pp. 35-40.
[4] Shekhter A.B., Lopatin V.V. Tissue reaction during polyacrylamide hydrogel implantation. The Reconstructive Surgery of the Mammary Gland Conference, Moscow, 1996, pp. 121-122. 